\title{
Therapeutic Use of Ultrasound Associated to Microbubbles in Acute Myocardial Infarction
}

\author{
André Luiz Cerqueira Almeida \\ Hospital Dom Pedro de Alcântara; Santa Casa de Misericórdia de Feira de Santana, Feira de Santana, BA - Brazil
}

This issue of Arquivos Brasileiros de Cardiologia Imagem Cardiovascular presents the important article "Ultrasound and microbubbles for coronary artery recanalization in acute myocardial infarction." 1 The first author of the study is Dr. Bruno Garcia Tavares and the last one is Dr. Wilson Mathias Jr., both from Instituto do Coração da Faculdade de Medicina da Universidade de São Paulo and Grupo Fleury, São Paulo, Brazil, with participation of Dr. Thomas Richard Porter, from the University of Nebraska. It is a pilot study that aimed to evaluate the effectiveness of intravenous microbubbles $(\mathrm{MB})$ and ultrasound (US), a procedure known as sonothrombolysis on the initial rates of recanalization of coronary arteries in patients with acute ST segment elevation myocardial infarction (STEMI). This study brings an auspicious message: the possibility of a therapeutic option that assists in the recanalization of coronary artery and consequent myocardial preservation in patients with STEMI.

The use of contrast agents in echocardiography (CAE) has shown significant progress. Microbubbles suspension created by manual agitation was initially used. ${ }^{2}$ These microbubbles, however, were large and unstable. In the evolution of the process, more stable microbubbles were created. These could pass through the pulmonary capillaries and reach the left heart chambers with a high degree of safety, reliability and diagnostic efficiency. ${ }^{3,4}$ In these days, the use of CAE is not limited to diagnosis, but also includes therapeutic support in many segments of medicine, including as an auxiliary therapy to thrombolysis. ${ }^{5,6}$

The mechanism by which the use of MB associated to US causes thrombolysis appears to be multifactorial. The most accepted one is the mechanical action. Specific ultrasonic pulses issued during off-the-shelf $\mathrm{MB}$ infusion cause the growth and collapse of microbubbles in the course of insonation, an effect known as cavitation. This transformation in the shape of microbubbles culminates in their rupture. This process causes shear stress in the areas surrounding the microbubbles. If this occurs in an area with thrombus, it will be achieved and eventually dissolved. ${ }^{7,8}$ Another mechanism of improved ischemia appears to be associated with the release of nitric

\section{Keywords}

Myocardial Infarction; Contrast Media; Microbubbles/ therapeutic use.

Corresponding author: André Luiz Cerqueira de Almeida •

Rua Alto do Paraguai, 280. Postal Code 44085-232, S.I.M.,

Feira de Santana, BA - Brazil

E-mail: andrealmeida@cardiol.br

DOI: $10.5935 / 2318-8219.20160021$ oxide. Specific ultrasonic pulses, when applied to the heart during infusion of $\mathrm{MB}$, causes the release of nitric oxide, which improves microvascular flow in ischemic regions and enhances the effects of thrombus dissolution caused by cavitation., ${ }^{9,10}$

Studies have shown that use of MB associated with US induces acceleration of thrombolysis during acute stroke, causing a more complete recanalization and a tendency to improve short and long-term outcomes in humans. ${ }^{5}$ Xie F. et al. showed that MB associated with US improves microvascular flow in the peri-infarcted region in pigs with STEMI. ${ }^{11}$

In the study by Tavares et al., ${ }^{1}$ the authors studied 24 patients in the acute phase of STEMI. These were randomized to three groups: the first one used $M B$, in continuous infusion, associated with US with mechanical index (MI) and high pulse duration between 4 and $20 \mu$ seg $(N=7)$; the second one, $\mathrm{MB}+\mathrm{US}$ with repetitive pulses of high $\mathrm{MI}$ with pulse duration $<2 \mu$ seg $(\mathrm{n}=8)$. All patients underwent coronary angioplasty $(\mathrm{PCl})$. In both groups, patients received therapy before and immediately after $\mathrm{PCl}$ for a total period of 50 to 60 minutes. Pre-PCl sonothrombolysis was conducted while the patient was waiting to be taken to the catheterization laboratory. The third group (control, $\mathrm{n}=9$ ) received $\mathrm{MB}$ and US with low $\mathrm{MI}$ only to analyze the perfusion inside the risk area at certain intervals. The controls also underwent PCI. The authors did not describe which other medications have been used by the patients. Angiography and echocardiography scans were conducted by experienced professionals blinded to the groups. Six out of 8 patients (75\%) of the group treated with MB and US with high and repetitive $\mathrm{MI}$ presented angiographic recanalization before angioplasty, compared with $43 \%$ (3 out of 7 patients) in the group receiving $\mathrm{MB}+\mathrm{US}$ with high $\mathrm{MI}$ and pulse duration between 4 and $20 \mu \mathrm{seg}$ and $11 \%$ (1 of 9 patients) in the control group. This difference in the angiographic recanalization rate was significant between the groups $(p<0.05)$. As an important fact, there was no difference in the door-to-balloon time between the three groups. Likewise, the duration of pain was similar between the groups.

The results of this study point to a major breakthrough in the treatment of patients with STEMI. Early recanalization data, formerly demonstrated in patients with stroke ${ }^{5}$ and in laboratory animals, are now reproduced in humans in the scenario of STEMI. ${ }^{1}$ This initiative, taken by the researchers, opens a great window of opportunity for further research with a larger number of patients randomized in clinical trials, adding knowledge in an area of global concern.

The study has some limitations: the number of patients studied was small, a fact recognized by the authors and understandable because it is a pilot study. Patients were recruited in a single center. The authors did not describe the risks and side effects associated with the therapy, if any, and also failed to describe 
the data associated with short and/or long-term evolution of the individuals in the study. Likewise, there was no description of hemodynamic abnormalities associated with the procedure. The same group of authors has recently published more robust data with a greater sample from the service, clarifying some issues listed as limitations in the current narrative. ${ }^{12}$

\section{References}

1. Tavares BG, Tsutsui JM, Aguiar MO, et al. Ultrassom e microbolhas na recanalização de artérias coronárias no infarto Agudo do Miocárdio. Arq Bras Cardiol: Imagem cardiovasc. 2016;29(2 supl 1):6.

2. Gramiak R, Shah PM. Echocardiography of the aortic root. Invest Radiol 1968;(5):356-66

3. Kurt M, Shaikh KA, Peterson L, Kurrelmeyer K, Shah G, Nagueh SF, et al. Impact of contrast echocardiography on evaluation of ventricular function and clinical management in a large prospective cohort. J Am Coll Cardiol. 2009;53(9):802-10.

4. Main ML, Hibberd MG, Ryan A, Lowe T, Miller P, Bhat G. Acute mortality in critically ill patients undergoing echocardiography with or without an ultrasound contrast agent. J Am Coll Cardiol Img. 2013;7(1):40-8.

5. Molina CA, Ribo M, Rubiera M, Montaner J, Santamarina E, Delgado -Mederos R, et al. Microbubble administration accelerates clot lysis during continuous 2-MHz ultrasound monitoring in stroke patients treated with intravenous tissue plasminogen activator. Stroke. 2006;37:425-9.

6. Tachibana K, Tachibana S. Albumin microbubble echo-contrast material as an enhancer for ultrasound accelerated thrombolysis. Circulation. 1995;92(5):1148-50
By achieving the proposed goals, the authors should be congratulated for the initiative and for developing this innovative and promising project. The scientific community will be awaiting further data that can prove the power of generalization and reproducibility of results, as well as more information about short and long-term safety offered to patients.

7. Xie F, Lof J, Everbach C, He A, Bennett R, Matsunaga T, et al. Treatment of acute intravascular thrombi with diagnostic ultrasound and intravenous microbubbles. J Am Coll Cardiol Img. 2009;2(4):511-8.

8. Xie F, GaoS, Wu J, Lof J, Radio S, Vignon F, etal. Diagnostic ultrasound induced inertial cavitation to non-invasively restore coronary and microvascular flow in acute myocardial infarction. PloS One. 2013;8(7):e69780.

9. Belcik JT, Mott BH, Xie A, Zhao Y, Kim S, Lindner NJ, et al. Augmentation of limb perfusion and reversal of tissue ischemia produces by ultrasound- mediated microbubble cavitation. Circ Cardiovasc Imaging 2015;8(4):pii:e002979.

10. Siegel RJ, Suchkova VN, Miyamoto T, Luo H, Baggs RB, Neuman Y, et al. Ultrasound energy improves myocardial perfusion in the presence of coronary occlusion. J Am Coll Cardiol. 2004;44(7):1454-8.

11. Xie F, Slikkerveer J, Gao S, Lof J, Kamp O, Unger E, et al. Coronary and microvascular thrombolysis with guided diagnostic ultrasound and microbubbles in acute ST segment elevation myocardial infarction. J Am Soc Echocardiogr. 2011;24(12):1400-8.

12. MathiasJrW, TsutsuiJM, Tavares BG, XieF, AguiarMO, Garcia DR, etal. Diagnostic ultrasound impulses improve microvascular flow in Patients With STEM Receiving Intravenous Microbubbles. J Am Coll Cardiol. 2016;67(21):2506-15 